\title{
A Comparative Analysis of GeneXpert Real-Time PCR with Culture for the Detection of Methicillin-Resistant Staphylococcus aureus Colonization in Selected Hospital Admissions
}

\author{
Fuzhan Parhizgar, ${ }^{1}$ Jane Colmer-Hamood, ${ }^{2}$ Jodene Satterwhite, ${ }^{3}$ \\ Richard Winn, ${ }^{1}$ and Kenneth Nugent ${ }^{1}$ \\ ${ }^{1}$ Department of Internal Medicine, Texas Tech University Health Sciences Center, School of Medicine, 36014 th Street, \\ Lubbock, TX 79430, USA \\ ${ }^{2}$ Department of Microbiology, Texas Tech University Health Sciences Center, School of Medicine, 3601 4th Street, \\ Lubbock, TX 79430, USA \\ ${ }^{3}$ Infection Prevention and Control, University Medical Center, Lubbock, TX 79430, USA
}

Correspondence should be addressed to Kenneth Nugent; kenneth.nugent@ttuhsc.edu

Received 10 August 2012; Accepted 13 September 2012

Academic Editors: J.-M. Bart, C. Ginevra, and M. Pourshafie

Copyright (C) 2013 Fuzhan Parhizgar et al. This is an open access article distributed under the Creative Commons Attribution License, which permits unrestricted use, distribution, and reproduction in any medium, provided the original work is properly cited.

Contact isolation of patients with methicillin-resistant Staphylococcus aureus (MRSA) reduces transmission to other patients and to health care workers. PCR technology can provide rapid detection of these patients. We tested the utility of using PCR for MRSA detection in patients with a history of MRSA infection or colonization or in a high risk group admitted to a general referral hospital. Nasal swabs from 342 patients were tested for MRSA on days one and three using the GeneXpert MRSA system. Swabs with a positive PCR result were cultured to identify staphylococcal species present in the nares. Fifty-six patients (38\% of 147) with a history of MRSA colonization or infection were positive; forty-seven patients (24\% of 195) in a high risk group were positive. Eighty-one percent of the patients with positive PCR swabs grew out MRSA on culture. Some cultures grew out only methicillinsensitive Staphylococcus aureus, methicillin-sensitive, coagulase negative Staphylococcus, or methicillin-resistant, coagulase negative Staphylococcus. This study demonstrates that most patients at risk for MRSA colonization are not colonized and that microbiological surveillance using PCR technology can facilitate contact isolation decisions. Not all PCR positive results represent the presence of MRSA, and hospitals need to consider policies for additional evaluation of positive PCR tests.

\section{Introduction}

Methicillin-resistant Staphylococcus aureus (MRSA) causes skin and soft tissue infections, sepsis, osteomyelitis, meningitis, endocarditis, urinary tract infections, necrotizing pneumonia, and toxic shock syndrome. These infections occur more frequently in men and in patients with significant comorbidity and increase hospital stays, mortality rates, and total costs [1]. In the early 1990s, hospitals started to use more stringent contact isolation guidelines for patients colonized or infected with MRSA to prevent the spread to other patients in the hospital [2]. However, many patients are not permanent carriers after infection or colonization and do not need full contact precautions and isolation upon readmission. Consequently, some hospitals now screen patients for MRSA infection upon admission, and these protocols range from patients in selected patient care units to universal screening of all patients $[3,4]$. We studied nasal colonization rates with MRSA in selected groups of patients rather than in all admissions or in particular patient care units to determine how useful faster diagnostic tests based on polymerase chain reaction (PCR) identification would be in screening these patients. 


\section{Methods}

The Infection Prevention and Control service at University Medical Center in Lubbock, TX, USA conducted a pilot project on the use of PCR screening for MRSA in June through August, 2010. Adult patients screened included those with a previous history of MRSA colonization or infection and those in high risk groups, including those in longterm care facilities, patients scheduled for CABG surgery, burn patients, and patients with trauma requiring intubation [2]. Nasal swab samples (Transystem LQ Stuart, COPAN Diagnostics, Inc., Murrieta, CA, USA) were taken from each patient upon admission and immediately sent to the laboratory for PCR testing using the real-time GeneXpert PCR assay (Cepheid, Sunnyvale, CA, USA). This assay tests for all the types of MRSA SCCmec A gene and has an average turnaround time of about seven hours [5]. When available, patients were also tested on the third hospital day. One deidentified sample from each patient with a positive PCR for MRSA was plated on tryptic soy agar (TSA) with 5\% sheep blood (BA) and provided to the research laboratory (Jane Colmer-Hamood) for bacteriological characterization. These specimens were incubated at $34^{\circ} \mathrm{C}$ for at least 48 hours. Typical staphylococcal colonies were subcultured on TSA without blood for pigmentation, BA for hemolysis, and mannitol salt agar and tested for the presence of clumping factor A and coagulase. Bacterial isolates with characteristic morphology which grew in $7.5 \%$ sodium chloride, used mannitol, and synthesized coagulase and clumping factor A were identified as Staphylococcus aureus. Bacterial isolates with characteristic morphology which grew in $7.5 \%$ sodium chloride, could not use mannitol, and did not make clumping factor A and coagulase were identified as coagulase negative Staphylococcus. All isolates identified as Staphylococcus were tested for resistance to oxacillin by spotting $25 \mu \mathrm{L}$ of a bacterial suspension in mueller hinton $(\mathrm{MH})$ broth to oxacillin screen agar $(\mathrm{MH}$ agar with $4 \%$ sodium chloride, $6 \mu \mathrm{g} / \mathrm{mL}$ oxacillin). The IRB at Texas Tech University Health Sciences Center approved the retrospective use of the results in this study.

\section{Results}

The clinical microbiology laboratory at University Medical Center conducted a pilot study before it introduced PCR technology for the identification of MRSA. This study included 107 specimens; 23 (21.5\%) were positive for MRSA either by PCR or culture or both. PCR tests had a sensitivity of $95.7 \%$ for identifying MRSA, a specificity of $100 \%$ for identifying MRSA negative specimens, and an overall accuracy of $99.1 \%$.

The screening project included 342 patients with 613 specimens (158 positive tests and 455 negative tests). One hundred and forty-seven patients had a history of MRSA colonization or infection, and 56 (38\%) had positive nasal screening using PCR (Table 1). One hundred and ninety-five patients had no history of MRSA colonization or infection but fell into high risk groups, and 47 (24\%) had a positive PCR screen. Thirty patients had two samples taken for testing, 18
TABLE 1: PCR results.

\begin{tabular}{lccc}
\hline & History of MRSA & High risk groups & Total \\
\hline No. of patients & 147 & 195 & 342 \\
No. of PCR positive & 56 & 47 & 103 \\
Percent positive & $38 \%^{* *}$ & $24 \%$ & $30 \%$ \\
\hline
\end{tabular}

** Percent positive significantly higher in patients with a history of MRSA infection or colonization than in patients in high risk groups.

$P<0.01$ by Chi square test.

had positive screens on both samples, and 12 had only one specimen positive. Nine patients had three samples taken for PCR testing. Seven were positive on all three samples, and 2 were positive on two of three samples.

One hundred and ten patients (103 in the table plus seven with second specimens) with a positive MRSA screens had positive bacterial cultures. Eighty-nine patients $(80.9 \%)$ had positive cultures for MRSA, 11 (10\%) had methicillinresistant, coagulase negative Staphylococcus, four $(3.6 \%)$ had methicillin-sensitive S. aureus (MSSA), five (4.5\%) had methicillin-sensitive, coagulase negative Staphylococcus, and one $(0.9 \%)$ did not grow out a staphylococcal isolate.

\section{Discussion}

Our study demonstrates that $38 \%$ of patients with a history of MRSA colonization or infection were nasal carriers upon readmission to the hospital and required contact isolation and that $24 \%$ of patients in a risk group were current carriers and required contact isolation. Therefore, these results demonstrate that microbiological surveillance with selective screening using rapid detection methods with PCR technology facilitates decisions regarding contact isolation. This approach should reduce transmission of MRSA from patients to healthcare workers and other patients and limit the use of unnecessary isolation. This approach should be more cost effective than using universal screening [4].

Our culture results indicate that not all patients with a positive PCR for MRSA had MRSA identified in nasal swab cultures. Some patients had only MSSA, methicillinresistant, coagulase negative Staphylococcus or methicillinsensitive, coagulase negative Staphylococcus recovered from nasal cultures. Therefore, these results indicate that PCR technology can result in misclassification using bacterial cultures as the gold standard. These false positive results may be explained by laboratory mistakes, the presence of nonviable bacteria on nasal swabs, and/or various genetic events which create discrepancies between PCR results and methicillin sensitivity [6-10]. These patients with a positive PCR test do need isolation. However, the next step in their management is uncertain. Sturenburg maintains that positive PCR results always require confirmation [9]. However, this approach would increase the cost and complexity of screening, and whether or not the correct identification of some hospitalized patients with staphylococcal colonization (but not MRSA) is important to discontinue contact isolation is uncertain. The answer to this question will likely depend on the prevalence 
of MRSA in the hospital, the patient mix, resources available for more testing, and overall infection control policies.

\section{Conflict of Interests}

The authors declare that they have no conflict of interests.

\section{Authors' Contribution}

F. Parhizgar collected the results, analyzed the results, and wrote the initial drafts of the paper. J. Colmer-Hamood collected the results, performed the bacteriological studies, and analyzed the results. J. Satterwhite helped design the study and collect the results. R. Winn helped design the study, analyze the results, and contributed to the paper preparation. K. Nugent did the IRB submission, analyzed the results, and prepared the final draft of the paper. All authors approved the final version.

\section{Acknowledgment}

This material was presented in an abstract form at the Southern Society of Clinical Investigation in New Orleans, LA, USA, in February 2011.

\section{References}

[1] G. A. Noskin, R. J. Rubin, J. J. Schentag et al., "The burden of Staphylococcus aureus infections on hospitals in the United States: an analysis of the 2000 and 2001 Nationwide Inpatient Sample database," Archives of Internal Medicine, vol. 165, no. 15, pp. 1756-1761, 2005.

[2] Center of Disease Control and Prevention, "2007 Guidelines for Isolation Precautions: Preventing Transmission of Infectious Agents in Healthcare Settings," http://www.cdc.gov/ ncidod/dhqp/pdf/guidelines/Isolation2007.pdf.

[3] M. C. Byrnes, T. Adegboyega, A. Riggle et al., "Nasal swabs collected routinely to screen for colonization by methicillinresistant Staphylococcus aureus in intensive care units are a sensitive screening test for the organism in clinical cultures," Surgical Infections, vol. 11, no. 6, pp. 511-515, 2010.

[4] N. Parvez, C. Jinadatha, R. Fader et al., "Universal MRSA nasal surveillance: characterization of outcomes at a tertiary care center and implications for infection control," Southern Medical Journal, vol. 103, no. 11, pp. 1084-1091, 2010.

[5] Cepheid, "Cepheid Xpert MRSA," 2010, http://www.cepheid .com/media/files/brochuresXpert\%20MRSA_V5_6pgr\%20US $\% 20$ Brochure.pdf.

[6] J. E. Corkill, J. J. Anson, P. Griffiths, and C. A. Hart, "Detection of elements of the staphylococcal cassette chromosome (SCC) in a methicillin-susceptible (mecA gene negative) homologue of a fucidin-resistant MRSA," Journal of Antimicrobial Chemotherapy, vol. 54, no. 1, pp. 229-231, 2004.

[7] C. Cuny and W. Witte, "PCR for the identification of methicillinresistant Staphylococcus aureus (MRSA) strains using a single primer pair specific for SCCmec elements and the neighbouring chromosome-borne orfX," Clinical Microbiology and Infection, vol. 11, no. 10, pp. 834-837, 2005.

[8] P. Francois, M. Bento, G. Renzi, S. Harbarth, D. Pittet, and J. Schrenzel, "Evaluation of three molecular assays for rapid identification of methicillin-resistant Staphylococcus aureus," Journal of Clinical Microbiology, vol. 45, no. 6, pp. 2011-2013, 2007.

[9] E. Stürenburg, "Rapid detection of methicillin-resistant Staphylococcus aureus directly from clinical samples: methods, effectiveness and cost considerations," German Medical Science, vol. 7, article Doc06, 2009.

[10] E. Creamer, A. Dolan, O. Sherlock et al., "The effect of rapid screening for methicillin-resistant Staphylococcus aureus (MRSA) on the identification and earlier isolation of MRSA- positive patients," Infection Control and Hospital Epidemiology, vol. 31, pp. 374-381, 2010. 


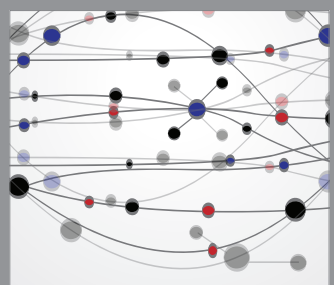

The Scientific World Journal
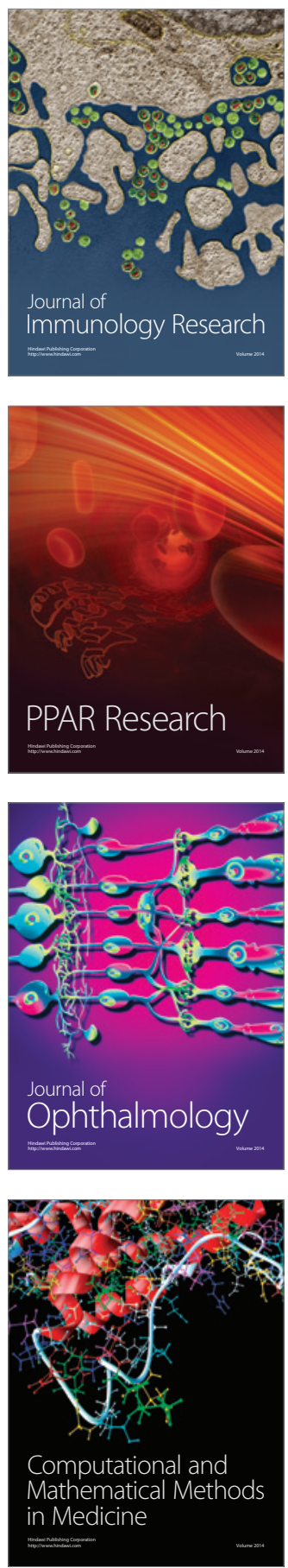

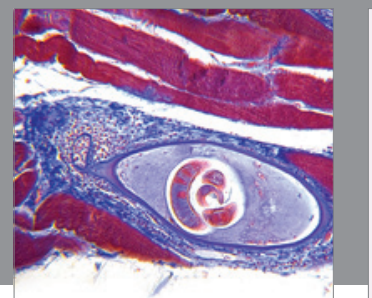

Gastroenterology

Research and Practice
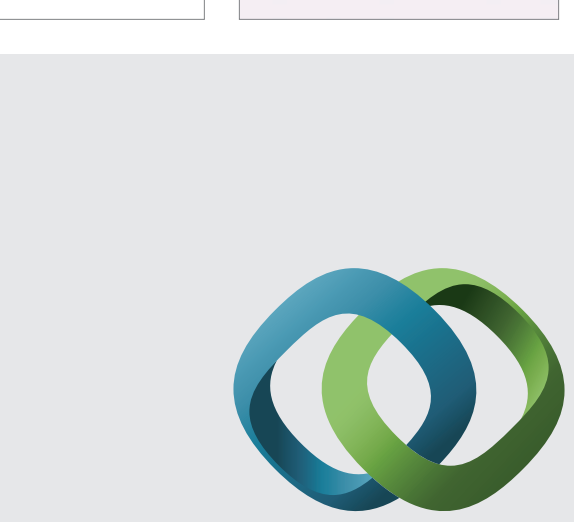

\section{Hindawi}

Submit your manuscripts at

http://www.hindawi.com
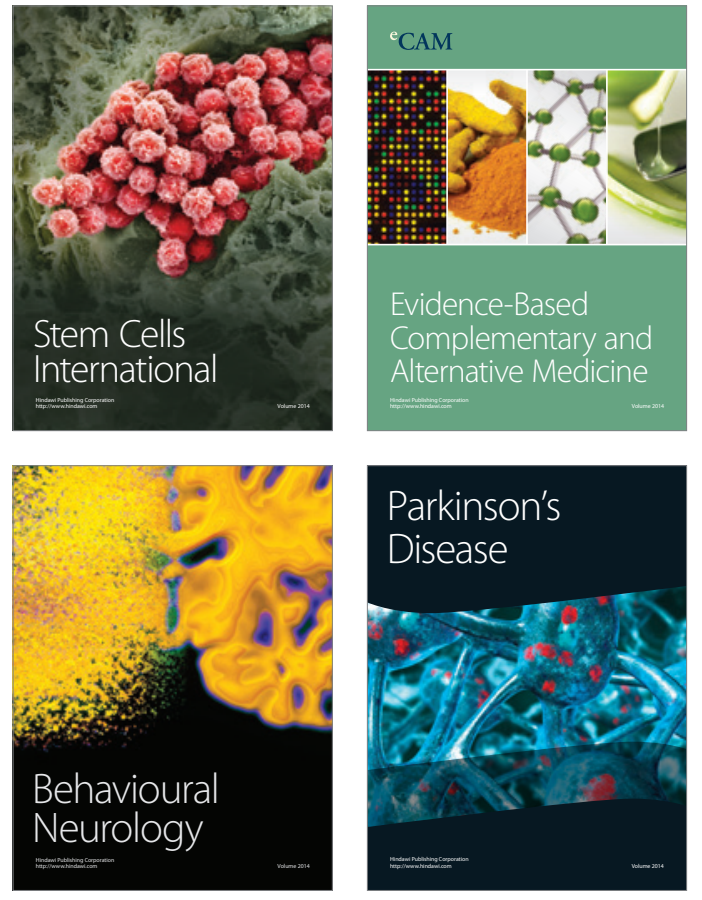
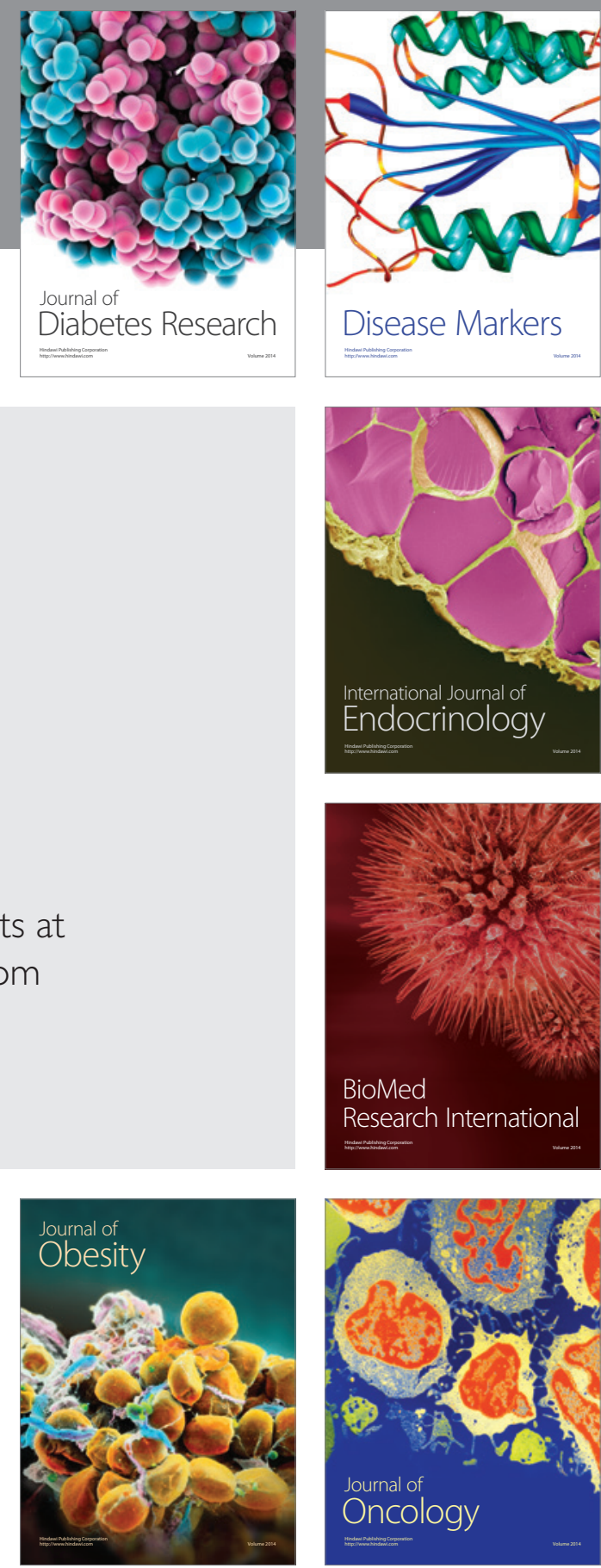

Disease Markers
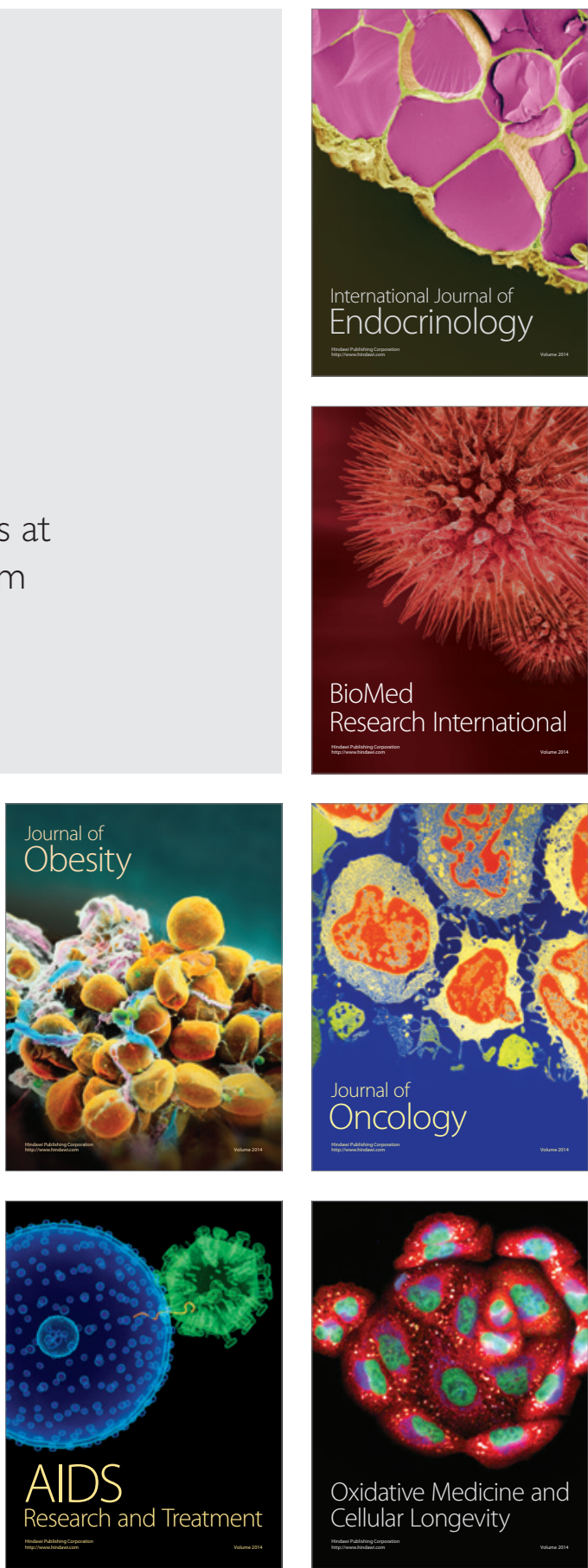